\title{
JOGOS EDUCATIVOS COMO OBJETOS DE APRENDIZAGEM PARA PESSOAS COM NECESSIDADES ESPECIAIS
}

Raquel Ribeiro Balbino - Depto de Ciências Exatas - Universidade Federal de Alfenas - raquelbalbino@gmail.com

Luiz Henrique Xavier Carneiro - Depto de Ciências Exatas - Universidade Federal de Alfenas - luizh_carneiro@yahoo.com.br

Mateus Drigo da Silva - Depto de Ciências Exatas - Universidade Federal de Alfenas mateus.drigo@gmail.com

Mariane Moreira de Souza - Depto de Ciências Exatas - Universidade Federal de Alfenas - mariane.souza@gmail.com

Melise Maria Veiga de Paula - Instituto de Ciências Exatas, Depto de Matemática e Computação - Universidade Federal de Itajubá - melisepaula@ gmail.com

Fabricia Ferreira de Souza - Programa de Pós Graduação em Ciência e Tecnologia da Computação - Universidade Federal de Itajubá - fabikk@ gmail.com

Resumo. O uso de jogos educativos se destaca como uma ferramenta bastante útil para o ensino/aprendizado no contexto de diversas áreas e públicos. No caso de pessoas com necessidades especiais, isso é especialmente importante, pois além de facilitar o aprendizado e motivar o aprendiz, contribui ainda para a inclusão digital deste tipo de público. Este artigo apresenta todas as atividades já desenvolvidas no processo de criação de um ambiente lúdico baseado em jogos para pessoas com necessidades especiais, envolvendo a realização de vários experimentos como auxílio no levantamento de requisitos para estes jogos.

Palavras-chave: jogos educativos, objetos de aprendizagem, levantamento de requisitos, personagens virtuais, necessidades especiais.

\begin{abstract}
Educative games as learning objects for peoples with special needs
The use of educative games stands out as a useful tool to teaching/learning in many areas and publics. In case of people with special needs this is very important, so besides motivates the students and facilitates the learning, it contributes to their digital inclusion. This article shows all the activities developed in the creation process of a ludic environment based in games for people with special needs, involving many experiments realization in order to help in the requirement specification process for this games.
\end{abstract}

Keywords: educative games, learning objects, requirement specification, virtual characters, special needs

\section{INTRODUÇÃO}

V. $7 \mathrm{~N}^{\circ}$ 3, dezembro, 2009 
Os jogos proporcionam desafios e ambientes visualmente atrativos, com a utilização de sons e imagens que motivam e estimulam o interesse, a atenção, a concentração e a memória do usuário. Com isso, eles deixaram de ser apenas instrumentos de entretenimento e passaram a se destacar como importantes ferramentas de apoio ao aprendizado, pois segundo Antunes (2000), o interesse do usuário passou a ser a força que comanda o processo de aprendizagem.

Alguns jogos possui características lúdicas, implicando não somente em um exercício cognitivo, mas principalmente, em um exercício afetivo. O lúdico ativa a imaginação pela busca de soluções. As situações imaginárias estimulam a inteligência e desenvolvem a criatividade (Almeida, 2006). Assim, os jogos se destacam como uma eficiente ferramenta pedagógica no aprendizado de pessoas com alguma deficiência mental que possuem dificuldades relacionadas à atenção e concentração, promovendo além da aprendizagem, inclusão digital e social.

Apesar de todas essas vantagens, a ausência de softwares educativos específicos para este tipo de público dificulta a utilização da abordagem de jogos para aprendizado neste contexto. Torna-se necessário, portanto, a criação de novos jogos, que considerem o perfil de pessoas com necessidades especiais, promovendo a melhoria do aprendizado das mesmas.

O objetivo do trabalho relacionado a este artigo é o desenvolvimento do ambiente virtual "Um Mundo Mais Que Especial" que deverá disponibilizar um conjunto de jogos educativos computacionais para facilitar o aprendizado de pessoas com necessidades especiais. Neste artigo, são apresentadas todas as atividades já desenvolvidas no processo de criação do ambiente mencionado, envolvendo a realização de experimentos em diferentes contextos e a elicitação de requisitos funcionais e não funcionais deste ambiente.

O artigo está organizado da seguinte maneira: a seção 2 apresenta a revisão de literatura relacionada à utilização de jogos como facilitadores do aprendizado. Na seção 3, é mostrado como jogos educativos podem ser utilizados como objetos de aprendizagem. Na seção 4, é apresentado o levantamento de requisitos do ambiente, bem como o contexto no qual estes requisitos foram identificados e são mostrados os resultados de um experimento realizado, verificando a importância de personagens virtuais em jogos voltados para pessoas com necessidades especiais. Finalmente, a seção 5 descreve o ambiente "Um Mundo Mais Que Especial" e os jogos que deverão ser disponibilizados num primeiro momento.

\section{O JOGO COMO SUPORTE AO APRENDIZADO}

A escola não é o único ambiente de aprendizado. Outros agentes como a comunidade, a interação com outros indivíduos e os meios de comunicação, também se apresentam como responsáveis pela formação e socialização das pessoas (Caetano, 2006). Os jogos computadorizados envolvem aspectos lúdicos, intelectuais, afetivos e sociais (Pinto et al., 2006). Neste contexto, eles surgem como uma forma de auxiliar os usuários no processo de ensino-aprendizado (Moraes et al., 2008b).

V. $7 \mathrm{~N}^{\mathrm{o}} 3$, dezembro, 2009 
Segundo Borges (2005), a pedagogia que utiliza o jogo como uma ferramenta de apoio ao processo de aprendizagem oferece algumas vantagens como ludicidade, cooperação, participação, prazer e motivação. E devido à grande aceitação deste tipo de ferramenta, principalmente pelo público jovem, a adoção dos jogos na área educacional representa um processo natural (Timm et al., 2008).

$\mathrm{Na}$ literatura, é possível encontrar uma grande variedade de jogos que tratam a questão do suporte ao aprendizado como, por exemplo, o jogo Gene2 que serve de apoio ao aprendizado de genética (Morelato et al., 2008), o projeto Saberlândia que visa desenvolver jogos educacionais voltados ao ensino fundamental (Pinto et al., 2006), o GeoplanoPEC, no ensino da geometria plana (Moraes et al., 2008a) e, no ensino de história, o jogo A Revolta da Cabanagem, que auxilia o aprendizado do período histórico da Cabanagem (Ribeiro Filho et al., 2006).

Contudo, Santaella (2004) afirma que o crescente avanço tecnológico da indústria de jogos acaba tornando-os cada vez mais complexos, exigindo, dentre outros, maior concentração e coordenação do usuário. Deste modo, pessoas com necessidades especiais, na maioria das vezes, não conseguem interagir com os jogos, causando assim desmotivação no aprendizado, além de problemas sociais (Fava, 2008). Isso demonstra a necessidade do desenvolvimento de aplicações específicas para esta classe de usuários.

Portanto, os jogos computacionais podem ser considerados uma alternativa legítima para atender as necessidades que envolvam o aprendizado. Mais especificamente, para pessoas com necessidades especiais, o apelo atrativo que permeia a utilização destas ferramentas através de um ambiente lúdico representa mais um fator que contribui ainda mais para sua utilização.

\section{JOGOS EDUCATIVOS COMO OBJETOS DE APRENDIZAGEM}

As inovações tecnológicas de recursos educativos digitais têm aumentado de forma considerável nos últimos anos e gerado discussões relevantes sob variadas perspectivas quanto à ampla possibilidade de uso dos objetos de aprendizagem. Dessa forma, os conceitos e regularização dos conteúdos digitais de aprendizagem passam a ser fundamentais para garantir aspectos como a interoperabilidade e reusabilidade dos mesmos (falta uma referencia aqui).

Segundo Wiley os objetos de aprendizagem (OA) são definidos como qualquer entidade, digital ou não digital, que pode ser utilizada, reutilizada ou referenciada durante o aprendizado suportado por tecnologias. Portanto, esses recursos tecnológicos podem ser considerados pequenas unidades de aprendizagem com possibilidades de serem usadas e reutilizadas em diversos contextos.

Dentre as diversas tecnologias que podem compor um objeto de aprendizagem, as mais utilizadas são: imagens, sons, animações, applets, documentos VRML e hipertextos. Não há limites para o uso desses recursos, porém, há um propósito em comum que deve ser respeitado: a construção do conhecimento através do estímulo da reflexão, habilidades e competências do aluno (Brasil, 2007, p. 20). 
A tendência em adaptar diferentes contextos de conteúdos às necessidades educativas do aluno tem gerado discussões sob diversos pontos de vista. Um exemplo é a padronização dos $\mathrm{OA}$, um assunto freqüentemente debatido em algumas instituições, tais como, a Advanced Distributed Learning (ADL) que teve a iniciativa de criar o modelo Scorm (Sharable Content Object Reference Model) (ADL, 2008), o IEEE (IEEE Standard for Learning Object Metadata - LOM) (IEE, 2002) e a ISO (Information Technology for Learning, Education and Training) (ISSO, 1988).

A padronização é sustentada por um conjunto de normas, especificações e orientações técnicas que visam garantir a qualidade dos objetos de aprendizagem sob diversos aspectos, os principais são:

- A durabilidade: capacidade para suportar os avanços tecnológicos sem custo de reconfiguração, replanejamento e recodificação.

- A interoperabilidade: habilidade de utilização em diferentes ferramentas ou plataformas tecnológicas.

- A reusabilidade: flexibilidade para incorporar componentes em múltiplas aplicações e contextos.

- A acessibilidade: refere-se a localização e acesso de um local remoto e usá-lo em muitos outros locais (Yokoyama e Carvalho, 2007, p. 3).

Recentemente, um aspecto importante que tem sido abordado no âmbito das tecnologias educacionais é a utilização de jogos educativos como objetos de aprendizagem. As atenções estão focadas em um modelo de aprendizagem efetivo que possibilita a representação e adaptação de jogos digitais como tecnologia educacional (Torrente et al., 2008).

Teixeira et al. (2007, p. 348) sugere a modelagem de jogos educacionais na forma de objetos de aprendizagem, com a finalidade de proporcionar recursos lúdicos e dinâmicos que apóiam o processo de aprendizagem com o uso do computador. Nesse sentido, o autor diz que a partir da associação entre os conceitos de jogos educacionais e objetos de aprendizagem origina-se o Objeto Jogo (OJ). Assim, todo objeto de aprendizagem formado por um único jogo é classificado como OJ simples, e quando o mesmo possuir outros elementos agregados como, texto, imagem, som, outros jogos, será considerado como OJ composto.

Levando em consideração todos os aspectos apresentados sobre o uso de jogos educativos como objetos de aprendizagem, as próximas seções abordam a proposta de desenvolvimento de dois jogos educativos para pessoas com necessidades educativas especiais, que representam os objetos de aprendizagem no contexto deste projeto.

\section{PROCESSO DE LEVANTAMENTO DE REQUISITOS}

Diante da dificuldade de comunicação com o público alvo que são os deficientes mentais, o processo de elicitação de requisitos exigiu a utilização de alguma estratégia que minimizasse essas dificuldades. Neste trabalho, a técnica de observação participante foi considerada por permitir um contato direto, frequente e prolongado com os usuários finais. Para isso, foi realizada a veiculação deste trabalho com um projeto de extensão 
da Universidade Federal de Alfenas em parceria com o Instituto Girassol. O Instituto Girassol é uma entidade sem fins lucrativos criada para desenvolver processos de inclusão social que envolvam pessoas com necessidades especiais.

O projeto de extensão conta com a participação de alunos com necessidades especiais do Instituto Girassol. Nas aulas, foram utilizados jogos de diversos temas e graus de dificuldade, sendo realizada a coleta de informações associadas às dificuldades do deficiente mental diante do computador. Dentre outros aspectos, são analisadas dificuldades motoras através de jogos que exigem o manuseio do mouse e teclado, dificuldades de atenção e concentração, o domínio de letras, sílabas e números e também o comportamento e convivência dessas pessoas com outras sem necessidades especiais.

A observação participante foi realizada durante aproximadamente 60 aulas de Inclusão Digital. As aulas são ministradas semanalmente desde março de 2008 por alunos do Curso Ciência da Computação sob a coordenação dos pesquisadores envolvidos. A duração de cada aula é de uma hora.

Inicialmente, foi realizada uma investigação no sentido de identificar ferramentas que poderiam ser utilizadas. Foram selecionados pacotes de jogos educacionais gratuitos e de diferentes contextos como o GCompris (GCompris, 2009), jogos da Turma da Mônica (Monica, 2009), dentre outros, para que os diferentes perfis de alunos pudessem ser atendidos.

A busca por ferramentas para uso nas aulas do projeto de extensão permitiu constatar o pequeno número de aplicações para este público específico. Os jogos encontrados até o momento, apesar de auxiliarem na questão da inclusão digital, apresentam falhas de usabilidade para este público e demonstram a necessidade do desenvolvimento de aplicações específicas para deficientes mentais.

Estas constatações foram validadas a partir de uma série de experimentos realizados, com a autorização dos responsáveis pelos alunos, durante as aulas do projeto de extensão. Durante os experimentos, após cada período de observação, os observadores elaboraram relatórios detalhando as principais constatações.

Outras técnicas de elicitação de requisitos utilizadas foram análise de documentos e entrevistas, nas quais foram consideradas as seguintes fontes de informação: pais ou responsáveis pelos alunos do Instituto Girassol, psicólogos, pedagogos e os demais professores do Instituto Girassol. Alguns documentos e relatórios do Instituto Girassol também foram analisados com o propósito de enriquecer as informações obtidas com as entrevistas.

A partir dessas técnicas foi feito o levantamento de requisitos, os quais estão listados abaixo juntamente com suas classificações. A seção 5 descreve o ambiente e os jogos que serão desenvolvidos a partir dos requisitos apresentados.

\subsection{Requisitos Funcionais}

Tabela 1 - Requisitos Funcionais 


\begin{tabular}{|c|c|}
\hline $\begin{array}{l}\text { [RF001] } \\
\text { Click com o } \\
\text { Mouse }\end{array}$ & $\begin{array}{l}\text { No jogo Pintando com Kadu, o sistema deve permitir que o usuário, } \\
\text { através de } 1 \text { click com o mouse, escolha a cor desejada na paleta e clique } \\
\text { novamente em cima do espaço que deseja colorir na figura. }\end{array}$ \\
\hline $\begin{array}{l}\text { [RF002] } \\
\text { Sons dos } \\
\text { animais }\end{array}$ & $\begin{array}{l}\text { No jogo Kadu no Zoológico, o sistema deve emitir o som dos animais que } \\
\text { estarão presos em alguma das jaulas. }\end{array}$ \\
\hline $\begin{array}{l}\text { [RF003] } \\
\text { Movimentar-se }\end{array}$ & $\begin{array}{l}\text { No jogo Kadu no Zoológico, o usuário terá a capacidade de movimentar- } \\
\text { se pelos labirintos. Usuário seleciona a }(s) \text { tecla }(s) \text { de movimentação. O } \\
\text { sistema realiza o movimento na direção informada pelo usuário. }\end{array}$ \\
\hline $\begin{array}{l}\text { [RF004] } \\
\text { Salvar }\end{array}$ & $\begin{array}{l}\text { Deverá ser possível que o ambiente, em qualquer momento, quando } \\
\text { requisitado pelo usuário, salve a fase atual em que o usuário está para } \\
\text { que ele possa posteriormente, voltar a jogar do ponto em que parou. } \\
\text { Consiste nos campos: nome e senha que devem ser preenchidos pelo(s) } \\
\text { tutor(es). }\end{array}$ \\
\hline $\begin{array}{l}\text { [RF005] } \\
\text { Cenários }\end{array}$ & $\begin{array}{l}\text { Os jogos devem utilizar cenários que reflitam imagens do cotidiano dos } \\
\text { usuários ou que sejam familiares ao ambiente. }\end{array}$ \\
\hline $\begin{array}{l}{[\mathbf{R F 0 0 6 ]}} \\
\text { Fases }\end{array}$ & $\begin{array}{l}\text { Os jogos devem apresentar diferentes fases para interação apresentando } \\
\text { graus variados de dificuldades. }\end{array}$ \\
\hline $\begin{array}{l}\text { [RF007] } \\
\text { Sequência }\end{array}$ & $\begin{array}{l}\text { Os jogos devem apresentar uma seqüência em relação às fases de forma a } \\
\text { facilitar a associação entre elas. }\end{array}$ \\
\hline $\begin{array}{c}\text { [RF008] } \\
\text { Menu Inicial }\end{array}$ & $\begin{array}{l}\text { Estando em qualquer momento de qualquer jogo, o ambiente deverá } \\
\text { permitir que o usuário volte à tela inicial da ferramenta, quando isso for } \\
\text { requisitado. }\end{array}$ \\
\hline $\begin{array}{c}\text { [RF009] } \\
\text { Abrir Fase }\end{array}$ & $\begin{array}{l}\text { O ambiente deverá solicitar o nome e senha do usuário para que possa ser } \\
\text { aberto o jogo na fase em que o usuário havia salvo. }\end{array}$ \\
\hline $\begin{array}{l}\text { [RF010] } \\
\text { Sair }\end{array}$ & $\begin{array}{l}\text { O ambiente deve permitir que o usuário saia do jogo atual a qualquer } \\
\text { momento. }\end{array}$ \\
\hline $\begin{array}{l}\text { [RF011] } \\
\text { Instruções }\end{array}$ & $\begin{array}{l}\text { Os jogos deverão apresentar a opção instruções que, através do } \\
\text { personagem virtual, explica o jogo para o usuário quantas vezes for } \\
\text { preciso. }\end{array}$ \\
\hline $\begin{array}{l}\text { [RF012] } \\
\text { Volume }\end{array}$ & $\begin{array}{l}\text { O ambiente deve apresentar a funcionalidade de controle de volume } \\
\text { (aumentar/diminuir), mas não deve permitir que o mesmo seja desativado. }\end{array}$ \\
\hline $\begin{array}{l}\text { [RF013] } \\
\text { Avisos }\end{array}$ & $\begin{array}{l}\text { O ambiente deverá emitir sons que indicam que o jogador fez alguma } \\
\text { escolha errada. }\end{array}$ \\
\hline $\begin{array}{l}\text { [RF014] } \\
\text { Alerta }\end{array}$ & $\begin{array}{l}\text { Se o usuário ficar mais de } 3 \text { minutos sem nenhuma interação com o jogo, } \\
\text { o personagem virtual o chamará para voltar a jogar. }\end{array}$ \\
\hline $\begin{array}{l}\text { [RF015] } \\
\text { Personagem } \\
\text { Virtual }\end{array}$ & $\begin{array}{l}\text { O ambiente educativo deve possuir um personagem virtual. No caso deste } \\
\text { ambiente, será um ratinho chamado Kadu que participará durante toda a } \\
\text { execução do ambiente. Ele tem como responsabilidade apresentar ao } \\
\text { usuário todos os jogos do ambiente; Depois de escolhido o jogo, é de sua } \\
\text { responsabilidade explicar o objetivo do jogo escolhido. Possui também a } \\
\text { função de motivar o jogador durante toda a partida, parabenizando-o em }\end{array}$ \\
\hline
\end{tabular}

V. $7 \mathrm{~N}^{\circ}$ 3, dezembro, 2009 
seus acertos e alertando-o com seus erros.

\subsection{Requisitos Não Funcionais}

\subsubsection{Usabilidade}

Tabela 2 - Requisitos Não Funcionais - Usabilidade

\begin{tabular}{|c|l|}
\hline $\begin{array}{c}\text { [RNF001] } \\
\text { Tarefas/Objetivos }\end{array}$ & $\begin{array}{l}\text { Os jogos devem ser fáceis de aprender e de usar. As tarefas a serem } \\
\text { realizadas e os objetivos devem ser explicados de maneira direta e } \\
\text { objetiva. }\end{array}$ \\
\hline $\begin{array}{c}\text { [RNF01tura, Cores e } \\
\text { Formas }\end{array}$ & $\begin{array}{l}\text { Os jogos não devem exigir leitura, nem reconhecimento de formas e } \\
\text { cores, mas associações. }\end{array}$ \\
\hline $\begin{array}{c}\text { [RNF003] } \\
\text { Periféricos }\end{array}$ & $\begin{array}{l}\text { Os jogos não devem exigir um manuseio fino de periféricos como } \\
\text { mouse e teclado. }\end{array}$ \\
\hline $\begin{array}{c}\text { [RNF004] } \\
\text { Ícones }\end{array}$ & $\begin{array}{l}\text { Os ícones presentes nos jogos devem ser de tamanho ampliado para } \\
\text { facilitar a manipulação dos mesmos. }\end{array}$ \\
\hline [RNF005] & $\begin{array}{l}\text { Os jogos devem ser executados exclusivamente no modo full-screen, } \\
\text { para que o usuário especial não tenha sua atenção dispersada por } \\
\text { outros aplicativos. }\end{array}$ \\
\hline [RNF006] & $\begin{array}{l}\text { Nos jogos em geral, qualquer função relacionada ao uso do mouse, } \\
\text { poderá ser realizada através de qualquer botão do mesmo. }\end{array}$ \\
\hline Mouse &
\end{tabular}

\subsubsection{Software}

Tabela 3: Requisitos Não Funcionais - Software

\begin{tabular}{|c|l|}
\hline [RNF007] & $\begin{array}{l}\text { O ambiente a ser utilizado para o desenvolvimento do ambiente será a } \\
\text { Ambiente de } \\
\text { plataforma JAVA integrada com Adobe Flash, com suporte ao SGBD } \\
\text { MySQL. }\end{array}$ \\
\hline
\end{tabular}

A partir dos requisitos levantados, as seguintes etapas futuras serão realizadas:

- Definição de novos requisitos para o software, com base na lista inicial de requisitos levantados, bem como a modelagem dos diagramas de casos de uso e de classes para estes requisitos;

- Construção do software em iterações, com a produção de protótipos dos jogos, com base nos requisitos e nos documentos de modelagem de classe detalhados.

- Realização de testes e experimentos com o software desenvolvido com os alunos do Instituto Girassol, procurando validar o software construído.

\subsection{Personagem Virtual: uma análise experimental}

Um dos requisitos indispensáveis de um jogo educativo é a criação de um personagem virtual. Atualmente, evidências científicas começam a apontar que a presença de um personagem em uma interface pode aumentar a confiança do usuário 
r

(Rickenberg e Reeves, 2000), permitindo uma melhoria no processo de interação do mesmo com o computador.

No caso deste trabalho, foi definido um personagem virtual, um ratinho chamado Kadu, que estará presente em todas as fases e terá várias funções, dentre elas: explicar o objetivo do jogo para o usuário quantas vezes forem necessárias, fazer alguma animação que chame a atenção do usuário se este estiver alguns minutos sem nenhuma interação com o jogo, parabenizá-lo quando algum objetivo for alcançado e encorajá-lo a cada falha cometida.

Visando verificar a necessidade de criação e utilização do personagem mencionado, foi realizado um experimento durante as aulas do projeto de extensão a fim de analisar a atenção, o interesse e a interação dos alunos com a presença do personagem virtual. Para esta avaliação, foram considerados dois jogos que utilizam personagens virtuais para explicar as instruções e interagir com os usuários durante a execução da ferramenta. Os aspectos analisados especificamente a partir de questionários foram:

- Atenção: análise da atenção dos usuários na explicação das instruções dos jogos através do personagem virtual.

- Interação: verificação da conduta dos usuários em seguir as explicações dos personagens virtuais.

- Interesse: análise do interesse dos alunos diante da presença do personagem virtual, verificando sua permanência no jogo até o término de seus objetivos.

Abaixo estão apresentados alguns resultados a partir das observações realizadas no experimento. Como apresentado na Figura 1, todos os alunos prestaram atenção na explicação dos personagens virtuais. Já a Figura 2, demonstra que a maioria dos alunos seguiram as explicações dos personagens virtuais. A figura 3 demonstra que a maioria dos alunos permaneceu no jogo até o término de seus objetivos.

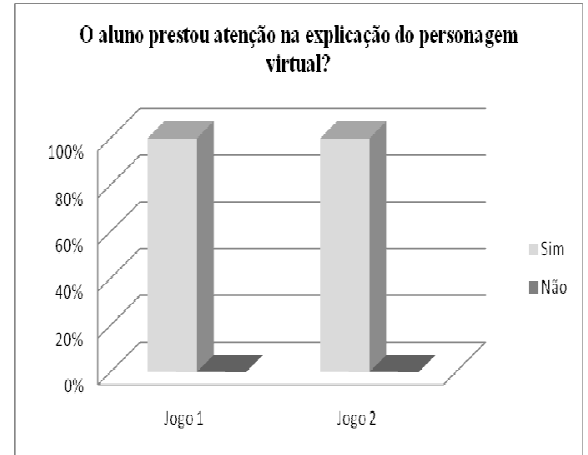

Figura 1 - Atenção

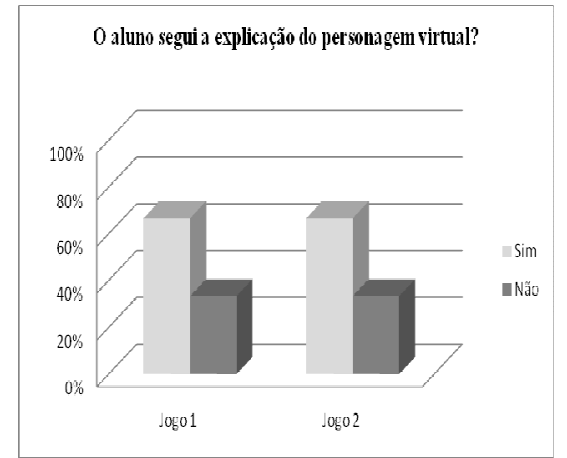

Figura 2 - Interação 
r

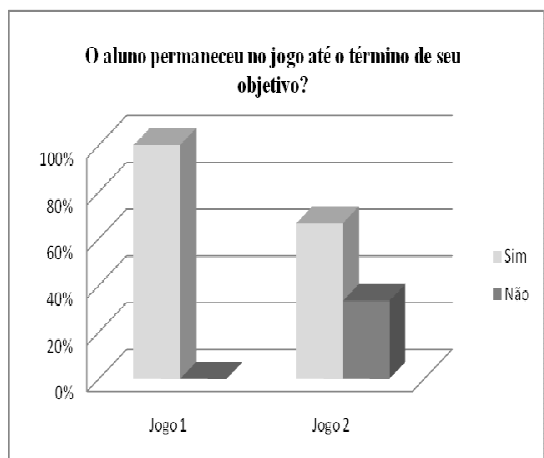

Figura 3 - Interesse

\section{O AMBIENTE "UM MUNDO MAIS QUE ESPECIAL"}

Abaixo estão descritos dois jogos que estão presentes no ambiente: Pintando com Kadu e Kadu no Zoológico.

\subsection{Pintando com Kadu}

O jogo é monousuário. Na tela, há o contorno de uma figura e a mesma em tamanho menor colorida no canto da tela. Abaixo, há o personagem virtual e uma paleta com as cores que o usuário precisará para colorir o contorno.

O objetivo do jogador é colorir o contorno da figura através de associações com a figura colorida, utilizando as cores presentes na paleta. Logo, o usuário não precisa reconhecer cores e formas e sim associá-las. O jogo é constituído de fases que vão aumentando o grau de dificuldade.

- $1^{a}$ fase: a figura é o cenário de uma feira de frutas com barracas contendo diversas frutas;

- $2^{a}$ fase: a figura é o cenário de uma paisagem de praia, com suas características específicas;

- $\quad 3^{a}$ fase: a figura é o cenário de uma paisagem de campo, também com suas características específicas;

- $4^{\mathrm{a}}$ fase: a figura é o cenário de um sítio com vários animais, típicos deste tipo de ambiente;

\subsection{Kadu no Zoológico}

O jogo é monousuário. Na tela é mostrado um zoológico com vários animais presos em jaulas e diversos labirintos até cada jaula. O jogo emite o som de um dos animais que estão presos. O objetivo do jogador é encontrar o caminho, através do labirinto, até a jaula do animal correspondente ao som emitido, usando as setas do teclado. A evolução das fases acontece a partir da existência de animais menos familiares ao jogador e labirintos mais complexos.

\section{REFERÊNCIAS}

V. $7 \mathrm{~N}^{\mathrm{o}}$ 3, dezembro, 2009 
ADL. Advanced Distributed Learning. ADL Guidelines for creating reusable content with Scorm 2004. July, 31, 2008. Version 1.0.

ALMEIDA, Anne. Ludicidade como instrumento pedagógico. 2006. Disponível em: $<$ http://www.cdof.com.br/recrea22.htm>. Acesso em: 11 set. 2009.

ANTUNES, Celso. Jogos para a estimulação das múltiplas inteligências. Rio de Janeiro: Vozes, 2000. p. 36.

BORGES, C. J. O Lúdico nas Interfaces das Relações Educativas. Revista de Pedagogia 12, Vol. 6, 2005, ISSN: 16779347.

BRASIL. Ministério da Educação. Secretaria de Educação a Distância. Objetos de aprendizagem: uma proposta de recurso pedagógico/Organização: Carmem Lúcia Prata, Anna Christina Aun de Azevedo Nascimento. Brasília: MEC, SEED, 2007. 154 p. ISBN: 978-85-296-0093-2.

CAETANO, K. C. Desenvolvimento e avaliação de um ambiente de aprendizagem virtual em administração em enfermagem. Dissertação da Universidade de São Paulo, Escola de Enfermagem, 2006.

FAVA, F. Jogando com o ar: o sopro como instrumento de acessibilidade nos jogos eletrônicos. In: SBGames 2008 - VII Symposium on Computer Games and Digital Entertainment, 2008, Belo Horizonte - MG, v. 1. p. 115-121.

GCOMPRIS, 2009. GCompris. Disponível em: <http://gcompris.net/-pt-br-.>. Acesso em: 04 jul. 2009.

IEEE. IEEE Learning Technology Standards Committee. Learning Object Metadata (LOM), 2002, Final Draft Standard, IEEE 1484.12.1.

ISO. International Standards Organization. "ISO Information Processing Systems Open Systems Interconnection - The Directory: Overview of Concepts, Models and Service.” ISO/IEC JTC 1/SC21; 1988. International Standard 9594-1.

MÔNICA. Portal da Turma da Mônica. Disponível em: <http://www.monica.com.br/>. Acesso em: 05 jul. 2009.

MORAES, D. B. S.; MORAES, M. B. S.; MACHADO, L. S.; REGO, R. G.; MORAES, R. M.; ANJOS, U. U. GeoplanoPEC: Um Jogo Inteligente Para o Ensino de Geometria Plana. In: SBGames 2008 - VII Symposium on Computer Games and Digital Entertainment, 2008, Belo Horizonte - MG, v. 1. p. 01-08.

MORAES, M. B. S.; DUTRA, D. L.; ANJOS, U. U.; REGO, R. G.; MORAES, R. M.; MACHADO, L. S. Geoplano: um jogo educacional inteligente para o ensino de geometria plana, 2008, INTERTECH - International Conference on Engineering and Technology Education, 2008, São Paulo.

V. $7 \mathrm{~N}^{\mathrm{o}}$ 3, dezembro, 2009 
MORELATO, L. A.; GUIMA, R. Y.; BORGES, M. A. F. Gene2: jogo via internet de apoio ao aprendizado de genética. In: SBIE 2008 - XIX Simpósio Brasileiro de Informática na Educação, 2008, Fortaleza - CE.

PINTO, I. M.; BOTELHO, S. C.; SOUZA, R. C.; GOULART, T. S.; COLARES, R.; CAMPOS, R. L. Saberlândia: plataforma lúdica integrando robótica e multimídia para educação. In: GT 2 - II Seminário Jogos eletrônicos, Educação e Comunicação, 2006, Salvador - BA.

RIBEIRO FILHO, M. R.; DAMASCENO, R.; REIS, F.; SILVA, F.; NASCIMENTO, M. Jogo Educativo Lúdico, A Revolta da Cabanagem. In: GT 2 - II Seminário Jogos eletrônicos, Educação e Comunicação, 2006, Salvador - BA.

RICKENBERG, R.; REEVES, B. The Effects of Animated Characters on Anxiety, Task Performance, and Evaluations of User Interfaces. In: Proceedings of CHI 2000 Human Factors in Computing Systems, 2000, The Hague, Amsterdam, The Netherlands.

SANTAELLA, Lucia. Games e comunidades virtuais, 2004. Disponível em: <http://www.canalcontemporaneo.art.br/tecnopoliticas/archives/000334.html>. Acesso em: 01 jul. 2009.

TEIXEIRA, J. S. F.; SÁ, E. J. V.; FERNANDES, C. T. Representação de Jogos Educacionais a partir do Modelo de Objetos de Aprendizagem. Instituto Tecnológico de Aeronáutica (ITA). Anais do XXVII congresso da SBC. WIE XII workshop sobre informática na escola. Rio de Janeiro, 2007.

TIMM, M. I.; RIBEIRO, L. O. M.; LANDO, V. R.; AZEVEDO, M. P.; VIEIRA, E. Game educacional: desafios da integração de elementos ficcionais, tecnológicos, cognitivos e de conteúdo. In: SBGames 2008 - VII Symposium on Computer Games and Digital Entertainment, 2008, Belo Horizonte - MG, v. 1. p. 107-114.

TORRENTE, J.; MORENO, P. G.; MANJON, B. F. Learning Models for the Integration of Adaptive Educational Games in Virtual Learning Environments. Dpto. Ingeniería del Software e Inteligencia Artificial. Facultad de Informática, Universidad Complutense de Madrid. Edutainment 2008, LNCS 5093, pp. 463-474, 2008 .

WILEY, D.A. Connecting learning objects to instructional design theory: A definition, a metaphor, and a taxonomy. Utah State University Digital Learning Environments Research Group. The Edumetrics Institute Emma Eccles Jones Education.

YOKOYAMA, B. P.; CARVALHO, S. T. Projeto e Implementação de Objetos de Aprendizagem SCORM. Instituto de Informática. Universidade Federal de Goiás. Março de 2007. 